\title{
Aproximaciones didácticas al concepto de estilo de enseñanza en docentes universitarios
}

\author{
Jaime A. Casas M. ${ }^{1}$ \\ Sandra R. Guáqueta P. ${ }^{2}$ \\ Artículo recibido el 10 de octubre de 2007 y aprobado el 27 de noviembre de 2008. \\ Didactic approaches to the concept of professors' \\ teaching style at the university
}

Resumen: En la determinación de la calidad del proceso de enseñanza de docentes universitarios intervienen una serie de factores que exigen una reflexión en torno a las prácticas de enseñanza, específicamente, en lo relacionado al estilo de enseñanza propio de cada profesor. En este artículo se identificaron los estilos de enseñanza de docentes de las carreras de Nutrición y Dietética y de Enfermería de la Pontificia Universidad Javeriana, Sede Bogotá, a partir de la concepción de estilo de enseñanza, definido en términos de particularidad y patrones de conducta del docente, que determina sus orientaciones y actitudes frente a ambientes disciplinares específicos, y respecto a su interacción con los estudiantes, en contextos determinados.

El estilo de enseñanza se caracterizó a partir de tres dimensiones: la dimensión personal, o también llamada teoría didáctica implícita, valorada desde tres categorías deductivas: comprensión de aprender, comprensión de enseñar; y comprensión de evaluar y desde tres categorías inductivas: valoración del estudiante, relación afectiva con el estudiante y percepción de compromiso con la labor docente. La dimensión metodológica, se valoró a partir de las categorías deductivas de planeación y desarrollo, y una categoría inductiva: motivación como elemento esencial en la vida del aula. Finalmente, la dimensión evaluativa, se valoró a partir de las categorías de estrategias, técnicas y recursos. Se concluyó que en algunos casos, pero no en todos ellos, existía coherencia de los estilos encontrados con el Proyecto Educativo Institucional, PEl, y con el currículo de la carrera estudiada. Una vez identificado el estilo del docente universitario, se propone una serie de lineamientos generales para la transformación de las prácticas de enseñanza, orientados a cubrir el espectro de calidad hacia el mejoramiento del desempeño.

Palabras clave: Estilo de enseñanza, prácticas de enseñanza y prácticas docentes.

Abstract:When determining the quality of the teaching process at the university there is a series of factors that demands a reflection around the educational practices, specifically, the issues related to professors' teaching style. In this article some teachers' styles are identified in two different carriers: Nutrition and Dietetic, and Nursing at Pontificia Universidad Javeriana, based on the conception of teaching style, defined in terms of particularity and professors ' behavior patterns, which determine their guidance and attitudes when facing the specific fields in regards to interaction with the students in specific contexts.

Key words:Teaching quality, teaching styles, teaching practice.

1 Profesor tiempo completo del Departamento de Química de la Universidad Pedagógica Nacional, Bogotá.

Correo electrónico: jcasas@pedagogica.edu.co

2 Profesora tiempo completo y dedicación exclusiva de la Facultad de Enfermería de la Universidad Nacional de Colombia, Sede Bogotá.Correo electrónico: srguaquetap@unal.edu.co 


\section{Referentes conceptuales}

Para identificar el estilo de enseñanza de los docentes universitarios de las carreras Nutrición y Dietética, y Enfermería, se partió de una revisión bibliográfica derivada de la conceptualización del estilo de enseñanza, en la cual se encontró que existe una gran diversidad de afirmaciones relacionadas con este concepto. Los autores de la investigación decidieron retomar los definidos por $\mathrm{E}$. Weber (1976): "Rasgo esencial, común y característico, referido a la manifestación peculiar del comportamiento y la actuación pedagógica de un educador o de un grupo de educadores que pertenece a una misma filosofía” y el definido por Beltrán et al, (1990): "ciertos patrones de conducta que el profesor sigue en el ejercicio de la enseñanza, iguales para con todos los alumnos y externamente visibles a cualquier observador".

Adicional a la definición recién descrita, existe otra que se complementa desde la acción misma de la manifestación en contextos áulicos: "Marca característica dada por una determinada manera de hacer-práctica que por consiguiente configura un particular modo de ser, una identidad, que a su vez, se despliega en los modos del hacer y allí es reconocida por otros" (Restrepo y Campo, 2002).

Respecto a la categorización de los estilos, específicamente, se presentaron diversas clasificaciones o tipologías, pero se retomaron en esencia las definidas por Palomino (2000) y Bennett (1979). Es así como se clasificaron los estilos de enseñanza según la perspectiva de Palomino, de acuerdo con la manera de concebir la tarea educativa, a saber, estilo técnico: integrado por profesores despreocupados del sentido y valor de su acción educativa- que se tornan transmisores de los contenidos en el plan de estudio, despreocupados de la calidad de la enseñanza, y que reproducen los estilos y modelos en que fueron instruidos. Estilo práctico: profesores que lo cuestionan todo, asumen su labor educativa con gran responsabilidad pretendiendo mejorar a los que les preocupa el porqué y el para qué de lo que hacen, siendo muy reflexivos en su labor. Estilo crítico: constituido por profesores muy reflexivos que entienden el desarrollo del currículum desde una comunidad educativa participativa y democrática, y que creen en la educación como medio de liberación y desarrollo humano.

Bennett (1979) argumenta sobre el hecho de que estas clasificaciones poseen una serie de vicios marcados por tres diversas clases de deficiencias, y es así como propone una tipología basada en doce estilos en el cual se alcanzan a esbozar tres grupos que colectan todos los estilos, en términos de los comportamientos instructivos y la gestión del aula, a saber: 1. Progresistas o liberales, con características tales como integración disciplinar, motivación intrínseca, agrupación flexible, que favorecen la elección de trabajos por parte del estudiante, y manifiestan cierta despreocupación por el control de la clase y el rendimiento. 2.Tradicionales o formales, caracterizados por poseer una motivación extrínseca, quienes casi no permiten elegir el tipo de trabajo al estudiante,por lo que agrupan a los estudiantes en forma fija (clase total y trabajo de corte individual), pero se preocupan por controlar el rendimiento escolar. 3. Estilos mixtos: situados entre 
uno y otro extremo, debido a la combinación en distintas proporciones de las dos categorías recién mencionadas. Respecto a las tipologías y actores seleccionados para la presente investigación, se puede afirmar que podría hacerse una equiparación, en términos generales, entre el estilo técnico de Palomino y los estilos tradicionales o formales de Bennett, así como son en buena medida equivalentes el estilo crítico de Palomino con los grupos de estilos progresistas o liberales de Bennett, aunque los definidos por Palomino se enfocan en la manera de interpretar la tarea educativa y los referidos por Bennett a la acción educativa propiamente dicha.

A partir del concepto mismo de estilo de enseñanza, se definieron tres dimensiones que permitieron enmarcarlo y abordarlo. 1.Denominada dimensión personal, o teoría didáctica implícita, valorada desde tres categorías deductivas (comprensión de aprender, comprensión de enseñar y comprensión de evaluar) y desde tres categorías inductivas, (generadas desde la presente investigación: apreciación y valoración del estudiante, relación afectiva con el estudiante y percepción de compromiso con la labor docente). 2.La segunda dimensión se denominó metodológica, valorada a partir de las categorías deductivas de planeación y desarrollo, así como una categoría inductiva, también generada desde el presente estudio: motivación como elemento esencial en la vida del aula. Finalmente, 3.La tercera dimensión es la evaluativa, valorada a partir de las categorías de estrategias, técnicas y recursos.

Por otra parte, se sustentó el presente trabajo en autores que profundizan en las dimensiones seleccionadas. En la dimensión personal, se emplearon los argumentos de Pérez Gómez (1984): "El pensamiento de los profesores orienta y dirige, aunque no de manera exclusiva su práctica profesional ",y de Rafael Porlán (1998) quien, citando a Polanyi (1967), afirma que este pensamiento se organiza en torno a esquemas de conocimiento que "suelen tener con frecuencia un carácter tácito y esto explica, en parte, que a veces puedan presentar contradicciones". En esta dimensión se considera la categoría de enseñar desde tres enfoques enunciados por Pérez Gómez (1999), uno en términos de transmisión cultural, uno más en términos del entrenamiento de habilidades y capacidades formales, y otro como el fomento del desarrollo natural; también se retoma la perspectiva de Freire (1997), quien considera que enseñar se refiere a crear las posibilidades para la propia producción o construcción.

Respecto a la categoría de comprensión de aprender, se utiliza la división de concepciones explicada por Gimeno Sacristán (1981), quien reconoce la existencia de diferentes teorías del aprendizaje: las teorías asociacionistas, las cuales consideran el aprendizaje como un proceso ciego y mecánico de asociación de estímulos-respuestas, y las teorías mediacionales, las cuales consideran que en todo aprendizaje intervienen, de forma más o menos decisiva, las peculiaridades de la estructura interna; siendo el aprendizaje un proceso de conocimiento, de comprensión de relaciones en el cual las condiciones externas actúan mediadas por las condiciones internas. Respecto a la categoría de comprensión de evaluar se fundamenta la presente investigación desde dos tendencias: 
una con planteamientos centrados en el incremento de la producción y otra que se expresa y concluye en "modelos" y "estrategias" de evaluación. Se sustenta la fundamentación respecto a esta categoría en lo afirmado por Torres (1999), quien explica la existencia de elementos coyunturales que amplían la complejidad de la comprensión de lo evaluativo como elemento central en la problemática educativa.

Respecto a la dimensión metodológica, se sustenta el trabajo en lo señalado por Gloria Edelstein (1996), quien afirma que existen dos posturas: una que "sacralizó el método", concibiéndolo como una serie de pasos, resultado de una suma de técnicas y procedimientos útiles en la solución de situaciones enmarcadas en ciertos contextos, los cuales explican la idea de un orden único y privilegian lo prescriptivo y lo normativo; regulando el quehacer del docente, quien se concibe como un experto en el manejo de la conducta. Y otra postura en la que el método se conforma en el marco de situaciones o ámbitos también particulares, dependiendo del contexto, según afirmaciones de Alicia de Camilloni (et al, 1996), quien considera que ésta relación está dada entre el contexto áulico social - cultural, lo epistemológico - objetivo y lo epistemológico - subjetivo. De igual manera, desde la óptica de Azucena Rodríguez y Gloria Edelstein (1996), ven el método, también, como un factor unificador de la propuesta docente e institucional.

Respecto a la dimensión evaluativa, se presentaron dos perspectivas: una como evaluación de productos, que considera como eje de la evaluación la determinación de objetivos y metas, al ser precisados como comportamiento observable, y otra en términos de evaluación de procesos, en la cual se tienen en cuenta las condiciones, para establecer medidas correctivas, si este fuera el caso. Por otra parte, la evaluación puede ser determinada como correspondiente a un determinado enfoque pedagógico, como lo afirma Flores Ochoa (1999), en el cual la evaluación es direccionada según el enfoque pedagógico, que puede ser tradicional, conductista, cognitivo o constructivista o social-cognitivo. Además, se tienen en cuenta las apreciaciones de Díaz Barriga (1998) al considerar los problemas y retos en el campo de la evaluación educativa.

\section{Metodología}

La investigación que se desarrolló es de tipo cualitativo, descriptivo-interpretativo y propositivo. Las fases fundamentales del diseño metodológico fueron:

\section{Fase 1.}

Selección de las profesiones y de la población objeto de estudio.

Se trabajó con las carreras Nutrición y Dietética y Enfermería de la Pontificia Universidad Javeriana, Sede Bogotá, y específicamente con la dirección de carrera, con los docentes y sus respectivos estudiantes.

La selección de los docentes de las carreras mencionadas se efectuó con base en un muestreo intencional no probabilístico, basado en criterios propios e intencionales, definidos por los investigadores, los cuales estaban centrados en:

- Experiencia como docentes universitarios de planta de la Pontificia Universidad Javeriana, por un periodo no menor de cinco años. 
- Valoración del desempeño catalogada como buena, con una ponderación superior al 70\% del total del puntaje posible en los formatos diligenciados por los estudiantes durante los dos últimos semestres.

- Y, finalmente, el concepto emitido por la dirección de carrera respectiva.

Por otra parte, se tomaron los estudiantes que estaban cursando estas asignaturas, en forma aleatoria y, cuando fue posible, se tomaron todos los estudiantes.

\section{Fase 2.}

Diseño de instrumentos y recolección de la información.

Basados en la revisión bibliográfica y con el objeto de diseñar los guiones de las entrevistas semiestructuradas (para directores de carrera y para profesores) o de grupo focal (para estudiantes), se seleccionaron las tres dimensiones desde las que se analizó el concepto de estilo de enseñanza: la dimensión personal, la dimensión metodológica y la dimensión evaluativa con sus respectivas categorías deductivas. Posteriormente, se realizó la validación de instrumentos, con base en el juicio de expertos y la prueba piloto. Una vez realizados los ajustes correspondientes, se realizaron las entrevistas respectivas a los ocho profesores (cuatro de Nutrición y Dietética y cuatro de Enfermería) a sus respectivos grupos de estudiantes y, además, a las dos directoras de carrera.

\section{Fase 3.}

Organización, sistematización, y análisis, de los datos

Durante esta etapa se transcribieron y codificaron las entrevistas realizadas. Para la interpretación y análisis se efectuó la validación de los datos cualitativos empleando la triangulación de la información obtenida. Para tal efecto, recogiendo las diversas miradas y perspectivas del estilo de enseñanza, se retomaron las conceptualizaciones de estilo de enseñanza propuestas por Weber (1976) y Beltrán (et al, 1990) por cuanto enmarcan los postulados emitidos por los otros autores, y por explicitar el estilo en términos de particularidad y patrón de conducta. Seguidamente se tipificaron y categorizaron los estilos de enseñanza de los docentes participantes en el estudio desde las dimensiones seleccionadas, así como desde las categorías de análisis, sean estas deductivas o generadas desde la investigación misma. Para explicitar en mayor grado de detalle, se presenta a continuación la secuenciación aplicada en esta fase del estudio. En primera instancia, se realizó el análisis del estilo de enseñanza derivado de la información obtenida de los docentes y de sus grupos de estudiantes, de las carreras Nutrición y Dietética y Enfermería. En segunda instancia, se realizó el análisis de la información derivada de los directores de carrera respectivos y, finalmente, se efectuó la identificación general del estilo definido por cada carrera, desde la definición de estilo de enseñanza generada en la investigación, y desde las tipologías definidas por los autores ya mencionados aplicando, para la validación de los datos cualitativos, la triangulación respectiva (De Tezanos, 1999). En todos los casos, se analizó la coherencia con el Proyecto Educativo Institucional de la Pontificia Universidad Javeriana y con el currículo de la carrera correspondiente y se aplicó el análisis a todas las categorías finalmente involucradas. 


\section{Resultados}

Con base en las respuestas generadas por los instrumentos, se presentan a continuación algunos apartes de las entrevistas que permiten consolidar los resultados en términos de dimensiones de análisis y categorías. Por ejemplo, con relación a la dimensión personal, relacionada con los implícitos o teoría didáctica implícita - en la cual surgieron una serie de categorías emergentes o inductivas - entre las cuales resaltamos la categoría de relación afectiva (a modo de ilustración, algunos apartes textuales de entrevistas de grupo focal, a estudiantes de Nutrición).

A continuación, reseñamos las convenciones empleadas en este estudio: $\mathrm{EN}=$ estudiantes de nutrición, $\mathrm{PN}=$ profesor de nutrición, $\mathrm{DE}=$ directora de enfermería.

Aquí, los estudiantes dan a conocer en sus respuestas una relación de proximidad afectiva que propicia responsabilidad y proyección en el estudiante al responder a la pregunta: ¿qué diferencia hay entre este docente de otros?:

EN1-2: [...] Yo creo que lo hace para que uno responda como estudiante, sí, porque si de pronto uno ve que una persona está pendiente de uno, ,uno cómo le va a faltar de pronto a esa persona, es que es más, [...] es como si uno le faltara a un amigo [...].

EN1-3: [...] Sí, uno se siente más comprometido con ella, [...] a mi personalmente no me gusta faltarle a ella porque, es tan buena, tan especial, el día que nadie lo haga yo no lo hago, porque el trato es más por su forma de ser.

En la categoría percepción de compromiso con la labor docente, los estudiantes EN1 dicen que ellos identifican en su do- cente, un gran sentido de responsabilidad frente al aprendizaje de sus estudiantes al responder a la pregunta: ¿cómo desarrolla el profesor sus clases?

EN1-2: [...] Ella como que está muy presente de cada estudiante y cuál es la falencia que uno tiene, entonces uno le pide la explicación y es como si le enseñaran qué es una suma: "una manzanita, más otra manzanita, nos da dos manzanitas", entonces ella piensa en dar una solución más fácil y mejor explicado que los otros profesores, entonces pues a mi me parece que así es ella [...].

En relación con la dimensión metodológica, se puede apreciar que se presenta, en uno de los casos, una contradicción entre lo expresado por el docente y el estudiante relacionado con la metodología utilizada para desarrollar sus prácticas de enseñanza:

El PN4 refiere en sus apreciaciones la manera en que prepara, planea y desarrolla sus clases a partir de la preparación de unas ayudas, unos cuestionamientos y ejercicios prácticos en los que le ofrezcan aplicabilidad a lo aprendido con relación en respuesta a la pregunta: ¿cómo prepara usted sus clases?

PN4: [...] Con base en mi programa, elaboro el tema, consulto lo último que hay, sobre todo en revistas y en libros, y también en relación a lo que hay en el medio para poder enriquecer al estudiante. [...] Traigo a colación el tema de la preparación de la clase, hago unas preguntas, para que pueda observar si realmente el estudiante se apropió del conocimiento que yo le di, que yo le estoy transmitiendo [...] llevo presentaciones [...] llevo casos de estudio y trabajos que se han elaborado para que las estudiantes los revisen y den sus propios conceptos, 
para empezar a definir criterios entre ellas $[\ldots]$.

[...] Yo les pregunto a las estudiantes si se acuerdan del tema anterior para poder hacer un vínculo con el tema que estamos dando. [...] Normalmente ellas tienen que haber leído sobre el tema que yo voy a tratar ese día. [...] Dependiendo de la metodología que yo voy a utilizar, entonces se empieza a trabajar el taller, o ellas empiezan a trabajar sobre las respuestas que ellas tienen; otra forma es que llego y les dicto el tema y después les hago unas preguntas de análisis acerca del tema que se vio, les dejo algún trabajo y se discute en la siguiente clase.

En contraste con lo anterior, los siguientes apartes presentan aseveraciones de los estudiantes que se contraponen a los expresados por la profesora; es así, como los estudiantes se refieren en respuesta a la pregunta ¿en cuanto a la preparación de las clases, qué nos pueden contar acerca del profesor?

EN4-3: [...] Ella se guía mucho de un libro, de un texto y, yo creo que esa es la vida de ella [...].

[...] La verdad es que lo que yo considero es que ella realmente no prepara mucho las clases. Ella a veces viene y nos cuenta algo, una parte teórica que ella ya sabe, y pues tantos años dándole a la misma materia, pues obviamente ya se lo aprendió [...].

Y además vamos un poquito atrasadas también, ella no lleva a cabo el cronograma, también nos manda tareas cuando tenemos prácticas, "no pues, niñas, averigüense el tema que hemos visto [...].

EN4-4: [...] Ella se para en el tablero y empieza a explicar unas cosas, experiencias, nosotras pues si de pronto tenemos dudas, le preguntamos
[...] y ya. En algunas ocasiones, ella nos asigna trabajos para realizar en grupo $[\ldots]$.

En relación con la dimensión evaluativa, y respecto a las categorías de estrategias, técnicas y recursos, DE afirma que la evaluación debe partir de lo que está establecido normativamente en la carrera de Enfermería, aunque el docente puede tener una cierta flexibilidad para realizar los ajustes, con el fin de poder identificar el progreso de sus estudiantes, como se interpreta en las siguientes respuestas con relación a esta pregunta: ¿cómo se debe evaluar en la carrera de Enfermería?

DE: [...] Desde el reglamento y desde el currículo se ha establecido un sistema de evaluación dependiendo del tipo de asignatura, si es sólo teórica, o si es teórico-practica; entonces ese es un elemento básico para determinar el sistema de evaluación. Pero también eso lo puede manejar, y lo debe manejar el profesor, quien es la persona que conoce exactamente el programa y sabe cuál es la mejor manera de evaluar esa área que él va a enseñar. Por lo que él puede determinar las diferentes formas de evaluación y el porcentaje que debe tener asignado cada una de esas posibilidades de evaluación. Pienso que de todas maneras la evaluación tiene que ser la posibilidad para el estudiante de saber cómo va en el logro de los objetivos propuestos, en qué tiene limitaciones y qué necesita para mejorar esos aspectos [...].

Se presenta a continuación la Tabla 1 , en la que se muestran en forma sintética las dimensiones y categorías evaluadas y se expresan las categorías emergentes que fueron generadas desde la presente investigación: 


\begin{tabular}{lll}
\hline Dimensiones & Categorías deductivas & Categorías inductivas o emergentes \\
\hline $\begin{array}{ll}\text { 1. Personal: implícitos } \\
\text { o teoría didáctica implícita }\end{array}$ & \begin{tabular}{l} 
Comprensión de enseñar \\
\cline { 2 - 2 }
\end{tabular} & $\begin{array}{l}\text { Caloración del estudiante } \\
\text { Relación afectiva con el estudiante } \\
\text { Percepción del compromiso con la labor } \\
\text { docente }\end{array}$ \\
\cline { 2 - 2 } 2. Metodolónica & Comprensión de evaluar & $\begin{array}{l}\text { Motivación como elemento esencial en } \\
\text { la vida del aula }\end{array}$ \\
\hline 3. Evaluativa & Desarrollo & \\
\hline
\end{tabular}

Tabla 1: Dimensiones y categorías evaluadas.

\section{Estilo de enseñanza general para la carrera de} Nutrición y Dietética

En términos generales, todos los profesores de la muestra se acomodan a la clasificación de tradicional o formal, según la tipología de Bennett (1979), mientras que, bajo la clasificación de Palomino (2000), algunos son de estilo técnico y otros de estilo práctico. Existe una amplia divergencia con el estilo de enseñanza propuesto por la Dirección de la Carrera, quien lo enmarcó en la clasificación de progresista o liberal, para Bennett (1979), y según Palomino (2000), práctico y crítico.

En casi todos los estilos determinados en el presente trabajo se encontró la preponderancia de la importancia de la interdisciplinariedad en el abordaje de las clases, pero existe una falta de concordancia parcial encontrada en el hecho de que algunos docentes fueron clasificados como técnicos, lo que está en franca contraposición con la proyección de los estudiantes hacia la formación integral del profesional, eje fundamental del PEI (Proyecto Educativo Institucional). Además, se evidenció que una de las docentes presenta contradicciones con lo expresado por sus estudiantes. Igualmente, todos los estilos de enseñanza son coherentes con el currículo de la carrera, ya sea en la capacidad de afrontar cambios en el conocimiento o en el desarrollo de acciones de nutrición, hacia el mejoramiento de condiciones de salud y nutrición en la población en el aspecto discursivo; además, hay una gran correspondencia con el estilo de enseñanza sugerido desde la Dirección de la Carrera.

\section{Estilo de enseñanza general para la carrera de Enfermería}

Todos los profesores de la muestra se clasifican como progresistas o liberales, según la tipología de Bennett (1979) como prácticos y críticos; según la propuesta por Palomino (2000) en completa concordancia con lo expresado por la Dirección de Carrera. En la carrera de Enfermería se encontró una articulación de todos los estilos de enseñanza con el PEI, siendo, así, que en todos ellos se busca la formación integral en los estudiantes, y en dos de los cuatro profesores de la muestra se encontró inclinación por el trabajo interdisciplinar. Por otra parte, la Dirección de Carrera apunta, además de la formación integral, a una visión contextual y al conocimiento de las tendencias de educación en enfermería, así como a un currículo basado en competencias cognoscitivas, comunica- 
tivas y socio-afectivas. La coherencia y articulación con el currículo de la carrera está fundamentada sustancialmente en el fomento del bienestar individual y colectivo, en el desarrollo de valores fundamentales para el cuidado de la salud, en la promoción y contextualización del estudiante, en todo caso en función de el cuidado de la vida misma; en este caso, la Dirección de Carrera apunta hacia la transformación de la realidad nacional y a la construcción de saberes propios del cuidado profesional de la vida desde la carrera de Enfermería.

Se presenta en las tablas 2 y 3 un resumen de los resultados obtenidos a partir del análisis de la información recabada para cada carrera estudiada.

Con base en los resultados generados, los cuales muestran estilos de enseñanza que no siempre corresponden con el currículo o con el PEI de la institución, en el cual, por ejemplo, aparecen estilos técnicos que se contraponen con la propuesta y la misión de la Universidad en términos de formación integral, pero también con base en la evidencia de las entrevistas en las que se pone de manifiesto un desconocimiento de las diversas perspectivas entre los actores del proceso educativo (estudiantes, profesores y directivas). Esta situación implica un problema de comunicación y de puesta en común, por tanto se presenta una serie de sugerencias y lineamientos para contribuir con la re-construcción de prácticas docentes que conduzcan a mejorar el desempeño hacia una docencia de calidad.

\section{Lineamientos generales para la re-construcción de prácticas docentes}

- Indagar sobre los implícitos de enseñantes antes del planteamiento o la aplicación de un proceso de formación de docentes es un paso previo y necesario para posibilitar la transformación de las prácticas docentes, debido a que, en la mayoría de los casos, es desde allí que las acciones docentes están fundamentadas.

- Un programa de formación de docentes debe contemplar: primero, el conocimiento de las percepciones de los actores, sean ellos directivos, profesores y estudiantes; y segundo, como su participación activa en este mismo programa, ya que no considerar una de estas miradas implica cambios parciales e incompletos.

- Es indispensable que todos los actores educativos de las diferentes carreras posean un conocimiento exhaustivo de los documentos institucionales, como el currículo, el plan de estudios, la misión y el PEI de la Universidad con miras a reorientar las acciones docentes hacia las intencionalidades y la propuesta formativa de la Pontificia Universidad Javeriana.

- Para alcanzar cambios profundos y completos hacia un proceso real y profundo de transformación y re-construcción de las prácticas de enseñanza, es imprescindible la integración de todo el cuerpo de profesores con el fin de evitar que las propuestas se queden en cambios puntuales de algunos de sus actores.

Cualquier programa o propuesta de re-construcción de las prácticas de enseñanza de los docentes debe surgir de una exploración previa de sus intereses, expectativas y necesidades; no siempre coincidentes con aquellas de los directivos.

- Para que todos los actores puedan acceder a la re-construcción y análisis de las prácticas docentes es imperativo que la institución genere y propicie espacios permanentes desde procesos secuenciales y graduales, pero no desde acciones aisladas y particulares. 


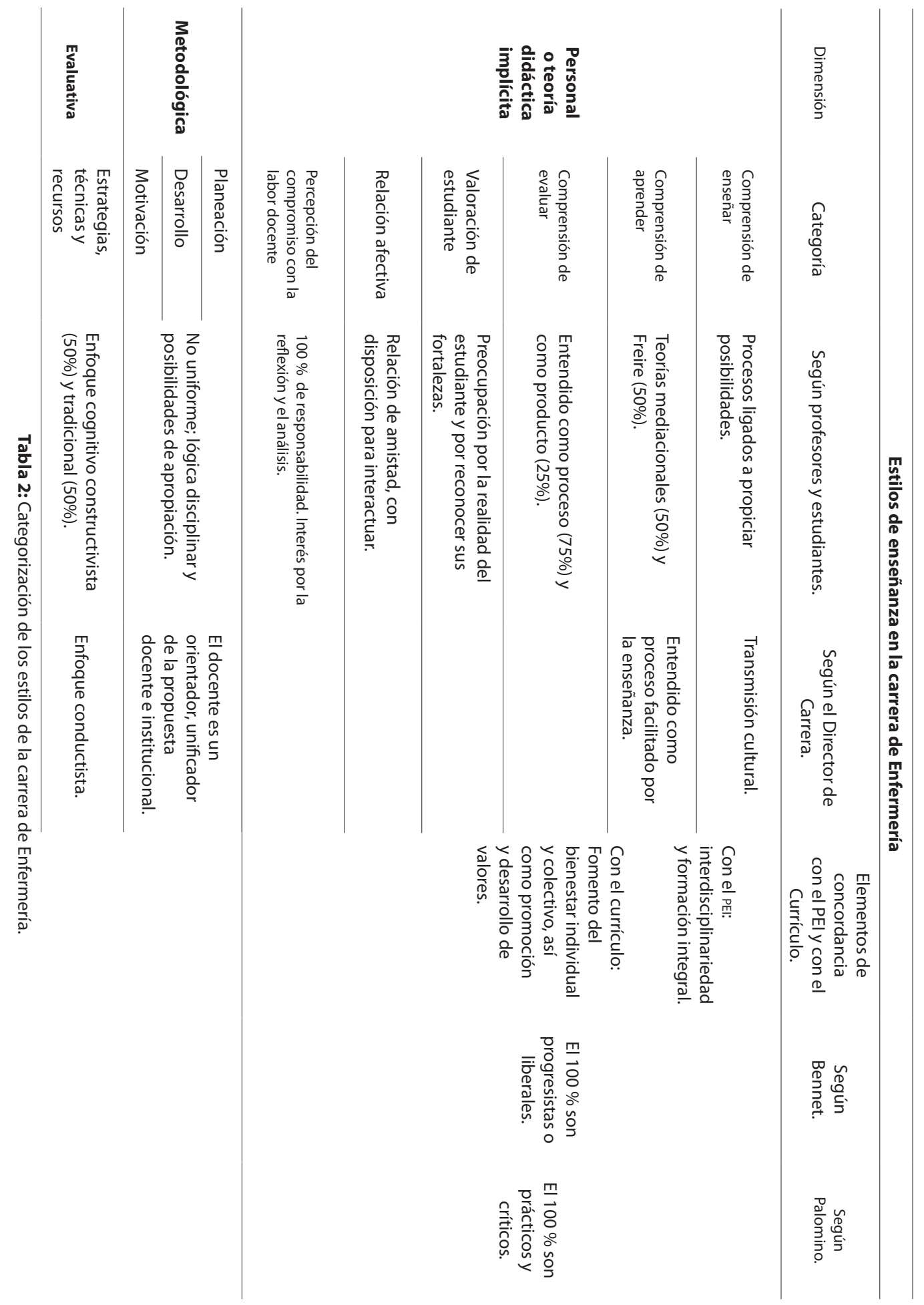




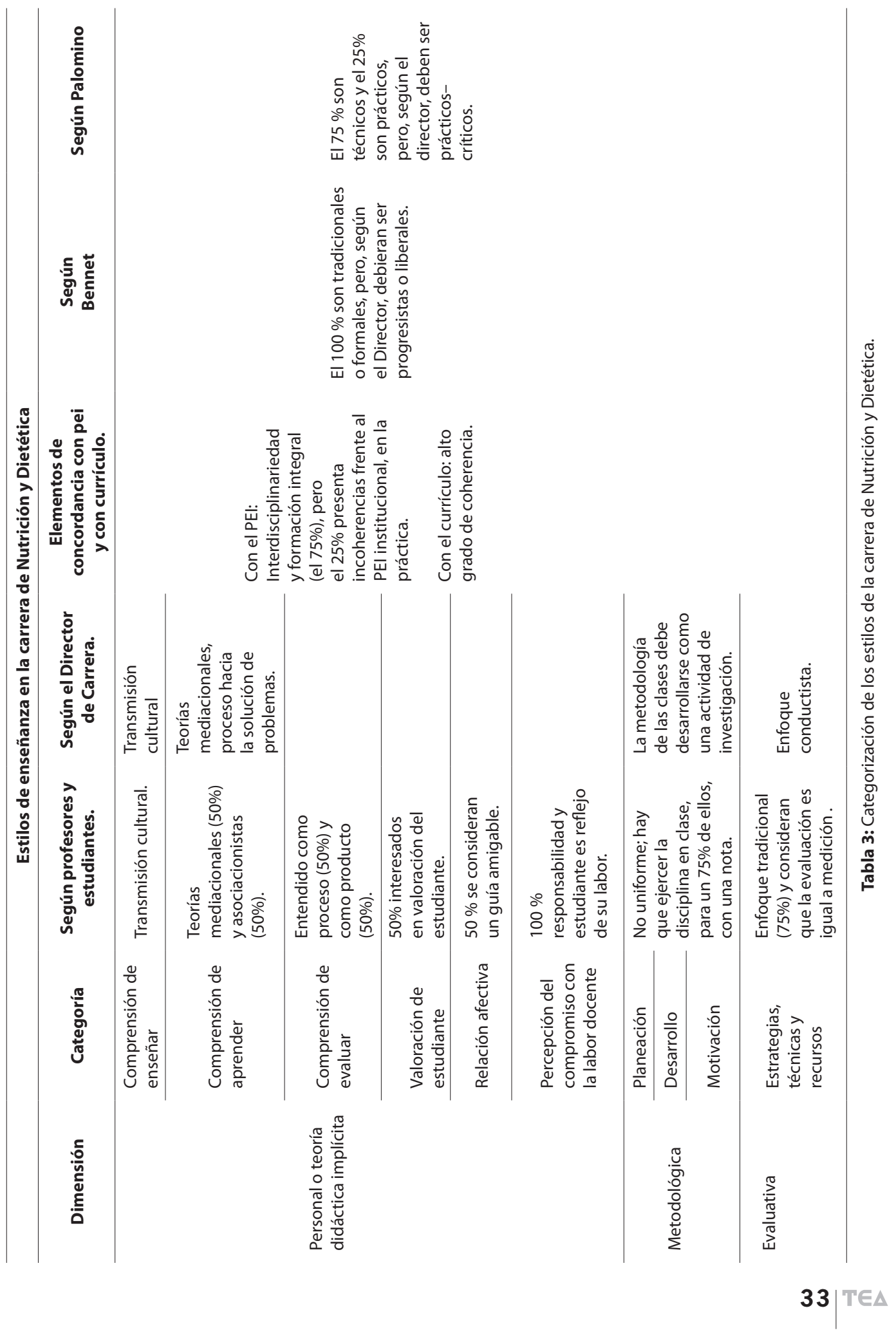


- Es desde la investigación y la reflexión epistemológica que se deben fundamentar las políticas y acciones de formación que generen una transformación real de las prácticas docentes y confluyan en las necesidades puntuales y específicas de la docencia universitaria.

- La implementación de cualquier tipo de transformación sobre el ejercicio de la práctica docente implica la consecución y asignación de recursos, además de un proceso de acompañamiento y apoyo permanente por parte de la Universidad como institución educativa.

- La socialización de resultados de investigación a toda la comunidad académica en relación con los procesos de cualificación docente es indispensable para la vinculación de todos los actores, para la generación de espacios de retroalimentación y para la consolidación de procesos cooperativos de mejoramiento de la docencia universitaria.

- La promoción y consolidación de espacios propios de divulgación de innovaciones y hallazgos en materia de pedagogía y didáctica fortalecen la investigación y favorecen el mejoramiento de las relaciones educativas en contextos académicos específicos.

\section{Conclusiones}

- El estilo de enseñanza de docentes universitarios, se puede definir en términos de la particularidad y los patrones de conducta del docente, los cuales determinan sus orientaciones y actitudes frente a sus ambientes disciplinares específicos y respecto a su interacción con los estudiantes, en contextos determinados.

- El estilo de enseñanza en docentes universitarios se puede caracterizar desde tres dimensiones: 1. La dimensión personal (que define la teoría didáctica implícita del docente) y la cual se puede valorar desde las categorías deductivas de comprensión de enseñar, comprensión de aprender y comprensión de eva- luar. 2. La dimensión metodológica, (que se puede valorar desde las categorías deductivas de planeación y desarrollo de sus clases) y, finalmente, 3. La dimensión evaluativa, que se puede valorar desde las categorías deductivas de estrategias, técnicas y recursos.

- Las categorías inductivas generadas a partir de esta investigación son: la apreciación y valoración del estudiante, la relación afectiva con el estudiante y la percepción del compromiso con la labor docente, pertenecientes a la dimensión personal, así como la categoría inductiva de motivación como elemento esencial en la vida del aula, ésta perteneciente a la dimensión metodológica, permitieron consolidar e integrar la caracterización del estilo del docente universitario de las carreras Nutrición y Dietética y Enfermería.

- Involucrar a los docentes y sus estudiantes respectivos como referentes válidos permitió generar múltiples perspectivas que favorecieron la explicitación del estilo de los docentes universitarios involucrados en la investigación.

- En términos generales, los estilos de enseñanza de los docentes de la carrera de Nutrición y Dietética, con respecto a su forma de concebir la tarea educativa, según Palomino (2000), se clasifican como técnicos (50\%) y prácticos (50\%); mientras que los docentes de la carrera de Enfermería (100\%) se encuentran en las tipologías tanto de prácticos como de críticos. Por otra parte, respecto a la gestión en el aula, según Bennett (1979), los profesores de Nutrición y Dietética se clasifican como tradicionales o formales (100\%), mientras que los profesores de la carrera de Enfermería, se clasifican como progresistas o liberales (100\%).

- Igualmente, en todos los estilos de enseñanza definidos se encontró que, en términos generales, respecto a la carrera de Nutrición y Dietética, existen elementos puntuales de coherencia con el PEI de la 
Pontificia Universidad Javeriana y, en algunos casos, elementos de incoherencia, que se observan por la diferencia entre la discursividad y la acción o por divergencias entre lo expresado por el profesor y lo expresado por los estudiantes, aunque se encontró un alto grado de coherencia con el currículo de la carrera. Respecto a la carrera de Enfermería se encontró una alta correspondencia y coherencia entre muchos rasgos y patrones de conducta del estilo de enseñanza con muchos elementos del PEI y también una muy alta correspondencia entre el estilo de enseñanza y el currículo de la carrera.

- El estilo de enseñanza desde los directores de carrera presenta una contribución en términos del currículo y está sustentado en procesos de indagación y búsqueda constructiva que, para la carrera de Nutrición y Dietética, equivale a investigación disciplinar.

- La clasificación del estilo determinado por estudiantes y profesores, en el caso de Nutrición y Dietética, difiere de aquella descrita por el Director; mientras que en el caso de Enfermería existe correspondencia entre lo mostrado por los profesores y el deber ser formulado por el Director de Carrera.

- Los estilos de enseñanza que favorecen actitudes positivas en los estudiantes frente al proceso de enseñanza son coherentes al considerar aprender, enseñar y evaluar como procesos ligados y en continua reconstrucción, además, involucran en el aspecto metodológico lo disciplinar, lo epistemológico subjetivo y el contexto áulico-social cultural.

- Información derivada de las categorías emergentes, tal como la percepción de inacabamiento, la de verse reflejado en sus alumnos y la de importancia del éxito académico en sus estudiantes son indicadores de estilos de enseñanza favorables para la promoción y proyección del entorno de aprendizaje.
- El proceso de investigación cualitativa fue enriquecedor en el sentido de propiciar otras miradas a la realidad, para generar una perspectiva más amplia a los investigadores del presente trabajo, para permitirnos proyectar nuestra calidad docente hacia la promoción de nuestros estudiantes y hacia nuestras propias prácticas de enseñanza.

- Las vivencias en el trabajo de investigación permiten consolidar las relaciones e interacción con otros maestros en la institución y abrir espacios hacia la construcción de programas de formación que confluyan en la integración y a la consolidación de equipos de mejoramiento pedagógico.

\section{Sugerencias}

- Realizar investigaciones similares en otras disciplinas y profesiones y ampliar el número de carreras estudiadas por Facultad con el fin de obtener un espectro más amplio en el cual se puedan hacer inferencias con mayor precisión.

- Desarrollar la investigación en otros niveles del sistema educativo (básica primaria o secundaria) o en instituciones de carácter técnico o tecnológico o en el nivel de posgrados (especializaciones, maestrías y doctorados).

- Estudiar la influencia del estilo de enseñanza del docente frente a rendimiento académico, deserción escolar, sentido de pertenencia, satisfacción con la institución en los estudiantes, etc.

- Estudiar la relación entre el estilo de enseñanza y el campo específico del conocimiento o el nivel en la carrera (básico o profesional).

\section{Referencias bibliográficas}

Beltrán, J., Moraleda. M. G., Alcañiz, E., G., Calleja, F. y Santiuste, V. (1990). Psicología de la educación. Madrid: Eudema Universidad Manuales. 
Bennet, N. (1979). Estilos de enseñanza y progreso de los alumnos. Madrid: Ediciones Morata.

Campo, R. y Restrepo, M. (1999). Formación integral: modalidad de educación posibilitadora de lo humano. Formas en Educación, 1.

De Camilloni, A., Davinci, M. C., Edelstein G., Litwin, E., Souto, M. y Barco, (1996). Corrientes didácticas contemporáneas. Buenos Aires: Editorial Paidós.

De Tezanos, A. (1999). Una etnografía de la etnografía. Bogotá: Ediciones Antropos.

Díaz Barriga, A. (1998). Problemas y retos del campo de la evaluación educativa, Investigador del Centro de Estudios sobre la Universidad. Perfiles Educativos, 37.

Edelstein, G. (1996). Corrientes didácticas contemporáneas. Argentina: Editorial Paidós.

Flórez Ochoa, R. (1999). Evaluación pedagógica y cognición. Bogotá: McGraw Hill.

Freire, P. (1997). Pedagogo de la autonomía. México: Siglo XXI Editores.

\section{Anexos}

Se presentan a continuación las guías de entrevistas aplicadas:

\section{Guía de entrevista aplicada a profesores}

La presente entrevista forma parte de un proyecto de investigación con el fin de identificar las principales características del estilo de enseñanza que emplea el docente en el desarrollo de sus prácticas. De antemano agradecemos su colaboración.
Gimeno Sacristán, J. (1981). Teoría de la enseñanza y desarrollo del currículo. Madrid: Anaya.

Palomino (2000) Algunos estilos docentes $y$ curriculares.

Porlán, R. (1998). Constructivismo y escuela. Hacia un modelo de enseñanza-aprendizaje basado en la investigación. Sevilla: Díada Editora.

Pontificia Universidad Javeriana. Proyecto Educativo Institucional. Bogotá: Pontificia Universidad Javeriana.

Restrepo, M. L. y Campo, R. (2002). La docencia como práctica. El concepto, un estilo, un modelo. Bogotá: Pontificia Universidad Javeriana.

Torres, G. (1999). De la evaluación del aprendizaje a la evaluación de los aprendizajes. Ponencia presentada en el Segundo Seminario Virtual: La Evaluación. Cededuis. Universidad Industrial de Santander, abril.

Weber, E. (1976). Estilos de educación. Barcelona: Herder.

Nombre:

Profesión:

Años de experiencia en docencia universitaria: 
Facultad a la que pertenece:

Asignatura que enseña:

e- mail:

Teléfono o extensión:

Fecha de la entrevista:

1. ¿Qué significa para usted enseñar?

2. ¿Qué es para usted el aprendizaje?

3. ¿Cómo prepara usted sus clases?

4. ¿Cómo desarrolla usted su clase?

5. ¿Qué es para usted la evaluación del aprendizaje?

6. ¿Cómo evalúa?

\section{Guía de entrevista aplicada a estudiantes}

Entrevista de grupo focal para aplicar a los estudiantes. El objetivo que tiene esta entrevista es conocer las principales características del estilo de enseñanza que emplea el docente universitario en el desarrollo de sus prácticas:

Estimados(a) estudiantes:

El objetivo de esta entrevista es conocer los principales elementos que, según su opinión, caracterizan el estilo de enseñanza del docente:

de la asignatura: La

información que ustedes nos proporcionen es confidencial y sólo será utilizada para el desarrollo de esta investigación. Agradecemos su colaboración.

Datos de identificación:
Nombres:

Facultad a la que pertenecen:

Semestre que cursan:

Fecha en que se realiza la entrevista

1. ¿Qué significa para ustedes enseñar?

2. ¿Qué significa para ustedes aprender?

3. ¿En cuanto a la preparación de las clases, qué nos pueden contar acerca del profesor?

4. ¿Cómo desarrolla su profesor las clases?

5. ¿Qué es para ustedes la evaluación del aprendizaje?

6. ¿Cómo los evalúa el docente?

7. ¿Qué diferencia a este docente de otros?

\section{Guía de entrevista aplicada a directores de carrera}

La presente entrevista forma parte de un proyecto de investigación con el fin de identificar las principales características del estilo de enseñanza que emplea el docente en el desarrollo de sus prácticas. De antemano agradecemos su colaboración.

Nombre:

Profesión: 
Años de experiencia en docencia universitaria:

Facultad a la que pertenece:

Asignatura que enseña:

e-mail:
Teléfono o extensión:

Fecha de la entrevista:

1. ¿Qué significa para usted enseñar?

2. ¿Qué significa para usted aprender?

3. ¿Qué elementos considera usted esenciales para la preparación de las clases?

4. ¿En la carrera de cómo deben desarrollarse las clases? 5. ¿Cómo se debe evaluar en la carrera de 